\title{
Practical Design of Dual Active Bridge Converter as Isolated Bi-directional Power Interface for Solid State Transformer Applications
}

\author{
Hyun-Jun Choi* and Jee-Hoon Jung ${ }^{\dagger}$
}

\begin{abstract}
As a bi-directional isolated interface for solid state transformer applications, practical design considerations of a dual active bridge (DAB) converter are proposed by means of a detailed mathematical model based on the converter's steady state operations. The DAB converter is useful in isolated bi-directional power conversion applications due to high performance, high efficiency, and bidirectional control manner. However, design considerations should be taken into account to overcome the disadvantages of DAB converter, such as low efficiency caused by restricted soft switching capability at light load conditions and high circulating current at heavy load conditions. The practical design considerations of the converter's power stage will be discussed to maximize soft switching capability and to minimize the conduction losses. In addition, to reduce the current stress of the power devices during a cold start sequence, an effective soft start algorithm will be proposed. Experimental results with a $3.3 \mathrm{~kW}$ prototype $\mathrm{DAB}$ converter will be given in order to validate the effectiveness of the proposed design considerations.
\end{abstract}

Keywords: Solid State Transformer (SST), Dual Active Bridge (DAB) Converter, Bi-directional power conversion, Soft start algorithm, Zero Voltage Switching (ZVS)

\section{Introduction}

Recently, over-dependency on the fossil fuels has created pollution, environmental damage, and, eventually, resource depletion problems. In addition, global warming is becoming a larger concern, and the electricity energy shortage problem can cause blackouts [1-3]. To address these problems, the demand for green energy continues to increase. Since green energy does not negatively influence the environment and uses renewable power sources, its application has become a significant research topic. Further, the smart-grid, micro/nano-grids and DC distribution systems are also critical issues in today's world market.

In particular, the solid state transformer (SST) has been actively studied, which is one of the key technologies for the smart-grid, micro/nano-grid, and DC distribution systems. As shown in Fig. 1, the SST is a bi-directional power conversion component that is used to interface a conventional AC grid with an AC/DC microgrid. Besides, the SST can be an alternative to substitute the oldfashioned line-frequency $(50 \mathrm{~Hz} / 60 \mathrm{~Hz})$ transformer [1, 4-5]. The weight and volume of the conventional transformers are heavy and bulky. Moreover, it is also a totally passive, causing reactive power. For these reasons, the applications of traditional transformers are very restrictive [6-8]. The

$\dagger$ Corresponding Author: School of Electrical and Computer Engineering, Ulsan National Institute of Science and Technology (UNIST), Korea. (jhjung@unist.ac.kr, jung.jeehoon@gmail.com)

* School of Electrical and Computer Engineering, Ulsan National Institute of Science and Technology (UNIST), Korea. (hyeonjun@unist.ac.kr)

Received: February 12, 2016; Accepted: April 24, 2016
SST can solve the problems of the line-frequency transformer by using a high frequency transformer with reactive power control. Furthermore, the SST has other advantages such as instantaneous voltage regulation, power factor correction, higher power density and systems protection capability. Fig. 2 shows that the SST consists of three parts: a bi-directional AC-DC rectifier, an isolated bi-directional DC-DC converter (IBDC), and a bidirectional DC-AC inverter.

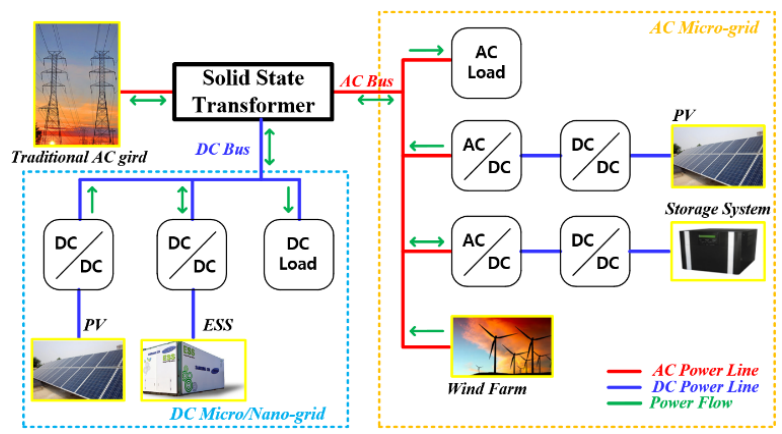

Fig. 1. Structure of AC/DC microgrid system based on SST.

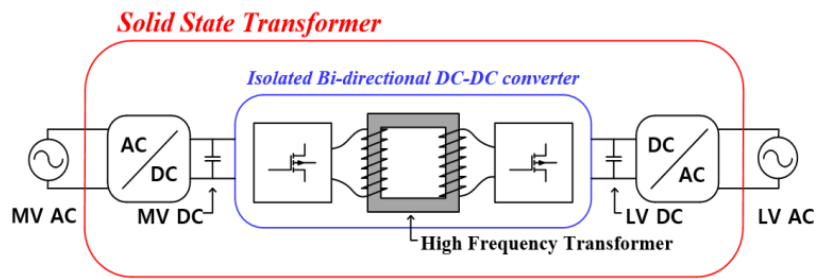

Fig. 2. Structure of SST with three power stages. 
The IBDC is one of the critical parts in the SST that is necessary to control the power flows and regulate the DC bus voltage. As one of based on isolated bi-directional bridge type topologies, the dual active bridge (DAB) converter has advantages, such as wide output voltage ranges and high efficiency. Particularly, based on a phaseshift modulation (PSM), the DAB converter can smoothly control the bi-directional power flows under a seamless voltage regulation manner. In addition, the DAB topology can reduce the switching losses by the inherent zero voltage switching (ZVS) capability. However, the DAB converter has disadvantage, such as high switching losses due to ZVS inadequacy at light load conditions and high conduction losses caused by high circulating current under heavy load conditions.

In this paper, to improve the performance of the DAB converter, a well-organized design methodology will be proposed. In Section II, operation principles of the DAB converter will be introduced briefly. In addition, practical design considerations of proper coupling inductance will be proposed to improve overall efficiency using RMS current analysis. To guarantee the maximum ZVS capability, effective dead time criterion will be determined based on a mathematical model. In Section III, a soft start algorithm will be proposed to suppress overcurrent stress of the power switches during the start-up sequence. Finally, in Section $\mathrm{IV}$, the proposed DAB converter design methodology will be verified by experimental data using a $3.3 \mathrm{~kW}$ prototype converter.

\section{Design Methodology}

Fig. 3 shows the schematic of the DAB converter. The $\mathrm{DAB}$ converter need to eliminate this parts, which is unnecessary consists of two single-phase symmetrical Hbridges with a coupling inductor. For the SST applications, a high frequency transformer with 1:1 turn-ratio is applied in the converter. For a simplicity in the converter analysis, the magnetizing current is ignored by assuming a perfect transformer.

\subsection{Operation principles of the DAB converter}

The operational principle of the DAB converter is

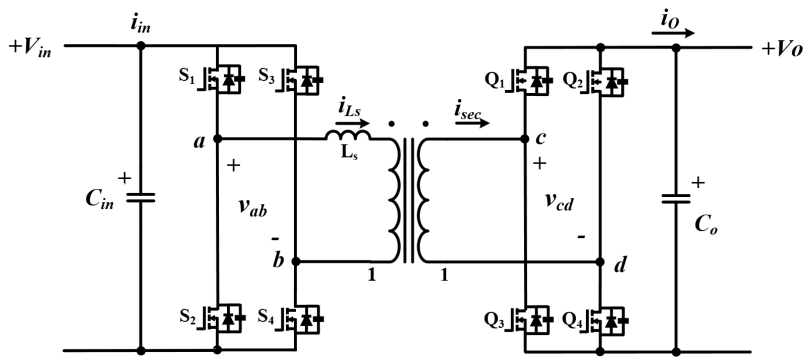

Fig. 3. Schematic of the DAB converter. simple and similar to the case of the phase-shift full bridge converter. As shown in Fig. 4, the DAB converter can achieve the ZVS when switches are turning on with sufficient negative primary current [9-11]. The steady state operation of the DAB converter is divided into two modes: forward and backward according to the current directions. Power is transferred from the leading bridge to the lagging bridge, and the two square waves are properly phase shifted to control the power flows. Output voltage can be regulated from the difference between the two H-bridge voltages, which is applied over the coupling inductance $\left(L_{S}\right)$ in the Fig. 3. The coupling inductance which is sum of the leakage inductance in the transformer and the external inductance acts as an energy transfer device [10-14].

\subsection{Coupling inductance current analysis}

Since the coupling inductance is a dominant parameter as the energy transfer component in the DAB converter, the analysis of the coupling inductor current becomes important in the operational principle of the DAB converter [10-12, 15]. Fig. 5 indicates the detail waveforms of the coupling inductor current, $i_{L s}$, in Fig. 4. From Fig. 5, each corner current $i_{1}$ and $i_{2}$ are derived as follows in (1) and (2):

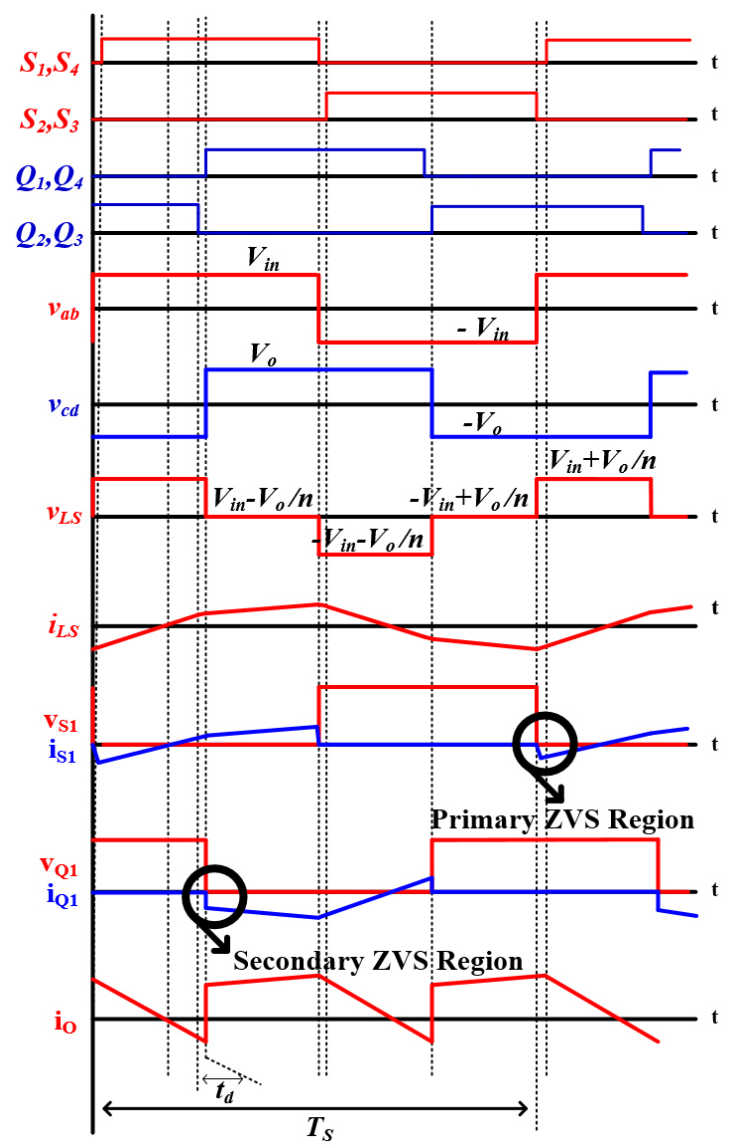

Fig. 4. Theoretical waveforms of the DAB converter in the forward power flow. 


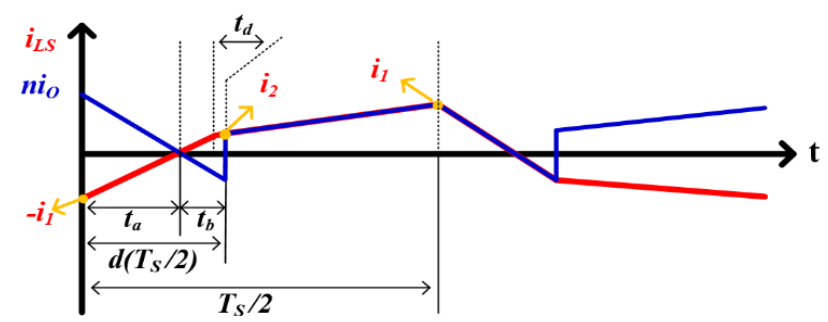

Fig. 5. Theoretical waveforms of the coupling inductor current and the average output current

$$
\begin{aligned}
& i_{1}=\frac{T_{S}}{4 L_{S}}\left[(2 d-1) \frac{v_{o}}{n}+v_{i n}\right] \\
& i_{2}=\frac{T_{S}}{4 L_{S}}\left[(2 d-1) v_{i n}+\frac{v_{o}}{n}\right]
\end{aligned}
$$

where $T_{S}$ is the switching period, $d$ is the amount of phaseshift radian/pi, $n$ is the turn ratio and $L_{S}$ is the coupling inductance. By using the corner values of the coupling inductor current, as shown in Fig. 5, the average input current, $I_{i n, a}$, and the average output current referred to the transformer primary side, $I_{o, a}$, can be derived as (3) and (4), respectively [12].

$$
\begin{aligned}
I_{\text {in }, a}(t)=\int_{\frac{T_{S}}{2}} i_{i n}(t) d t & =\frac{2}{T_{S}}\left[-\frac{1}{2} i_{1} t_{a}+\frac{1}{2} i_{2} t_{b}+\frac{1}{2}\left(i_{1}+i_{2}\right)(1-d) \frac{2}{T_{S}}\right] \\
& =\frac{V_{O} d(1-d) T_{S}}{2 n L_{S}} \\
I_{\mathrm{o}, a}(t)=\int_{\frac{T_{S}}{2}} n i_{o}(t) d t & =\frac{2}{T_{S}}\left[\frac{1}{2} i_{1} t_{a}-\frac{1}{2} i_{2} t_{b}+\frac{1}{2}\left(i_{1}+i_{2}\right)(1-d) \frac{2}{T_{S}}\right] \\
& =\frac{V_{i n} d(1-d) T_{S}}{2 n L_{S}}
\end{aligned}
$$

Theoretically, from (3) and (4), the maximum output power of the DAB converter can be obtained at $50 \%$ phaseshift between the two H-bridges; however, proper dead time should be considered for the ZVS conditions of the power switches and preventing the shoot-through faults. From (1) to (4), the optimal value of the coupling inductance, $L_{S}$, and the proper value of the dead time duration, $t_{d}$, can be derived.

\subsection{Proper design of coupling inductance}

The output power should be considered to design the proper coupling inductance. From (3) and (4), the gain formula and the output power of the DAB converter can be expressed as (5) and (6), respectively:

$$
M=\frac{V_{o}}{n V_{i n}}=\frac{I_{\mathrm{o}, a} R}{n V_{i n}}=\frac{T_{S} R d(1-d)}{2 n^{2} L_{S}}=k(1-d) d
$$

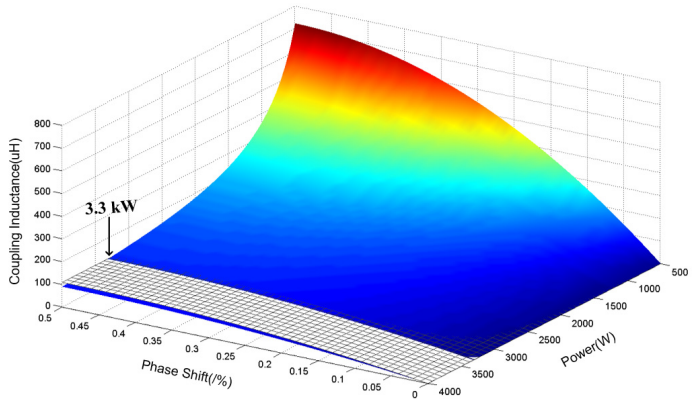

Fig. 6. Coupling inductance graph between the transfer power and the phase-shift

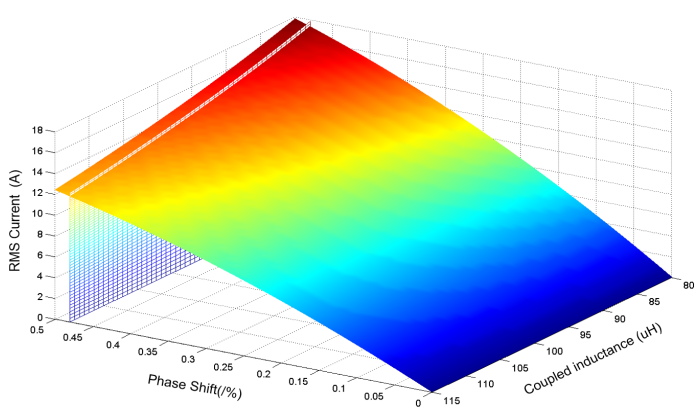

Fig. 7. RMS current graph between the coupling inductance and the phase-shift

$$
P_{\text {out }}=k V_{\text {in }} V_{o}(1-d) d \frac{n}{R}
$$

where $R$ is the load resistance and $k$ is defined in (7).

$$
k=\frac{T_{S} R}{2 n^{2} L_{s}}=\frac{P_{o u t}}{v_{o} v_{\text {in }}(1-d) d \frac{n}{R}}
$$

Using (5), (6), and (7), a proper design criterion of the coupling inductance can be derived as follows:

$$
L_{S} \leq \frac{V_{i n} d_{\max }\left(1-d_{\max }\right) T_{S} R_{\min }}{2 n V_{o}}
$$

where the $d_{\max }$ is the maximum phase gap between primary and secondary legs and $R_{\text {min }}$ is the minimum load resistance.

Fig. 6 illustrates coupling inductance variations according to the maximum power transfer and the amount of the phase-shift. It shows that smaller coupling inductance can transfer the higher power in the DAB converter. However, the smaller coupling inductance also can induce the higher conduction losses due to the higher RMS current expressed as shown in (9).

$$
I_{R M S}=\frac{T_{S}}{8 L_{S}\left(v_{i n}-\frac{v_{o}}{n}\right)}\left[\left(v_{i n}-\frac{v_{o}}{n}\right)^{2}+\frac{4 v_{i n} v_{o} d(2-d)}{n}\right]
$$


Fig. 7 shows RMS current variation according to the coupling inductance and the amount of the phase-shift. The RMS current is inversely proportional to the coupling inductance. Therefore, considering (8) and (9), a proper coupling inductance value should be determined.

\subsection{Dead time duration for soft switching}

One of design difficulties of the DAB converter is low efficiency under light load conditions caused by limited ZVS capability. This induces hard switching of the power switches, which reduces the overall power conversion efficiency. To ensure the maximum ZVS region, sufficient time for charge and discharge process of the power MOSFETs' output capacitance should be guaranteed, which requires an optimal dead time duration. In previous research, traditional dead time duration has already been derived as a necessary condition; however, they did not consider charging and discharging speed of the output capacitance [13-15].

Since the dead time should be longer than the charging and discharging duration of the MOSFETs' output capacitance, the dead time condition can be derived as follows [16]:

$$
4 C_{p} v_{\max } \leq i_{c} t_{d} \cong \min \left(i_{1}, i_{2}\right) t_{d}
$$

where $v_{\max }$ is the maximum voltage applied to the switches, $C_{p}$ is the output capacitance, $i_{c}$ is the current passing through the output capacitors, and $i_{1}$ and $i_{2}$ are the corner values of the coupling inductor current in Fig. 5. It is assumed that $i_{c}$ is constant during the dead time duration which is short enough. Consequently, the ZVS of the power MOSFETs can be guaranteed by the accurate dead time duration described as follows:

$$
t_{d} \geq 16 C_{p} L_{S} \frac{f_{S}}{M\left(1-d_{\max }\right)+1}
$$

where $f_{s}$ is the switching frequency.

\section{Soft Start Algorithm}

A conventional control method of the DAB converter uses the fixed duty ratio with the consideration of dead time which is applied to each power switch. The phase of the pulse width modulated (PWM) signal is shifted between the primary and secondary sides to regulate the output voltage. This control manner is so-called the PSM $[10-11,14]$. However, this output voltage control strategy is limited to only the steady-state operation of the converter. During a cold start sequence, the PSM directly transfers electric power from the primary side to the output capacitors. It causes inrush current due to rapidly charging

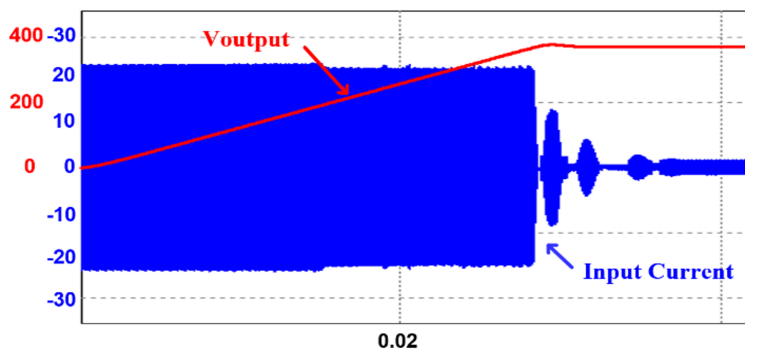

Fig. 8. Simulated inrush current of the DAB converter at the cold start duration

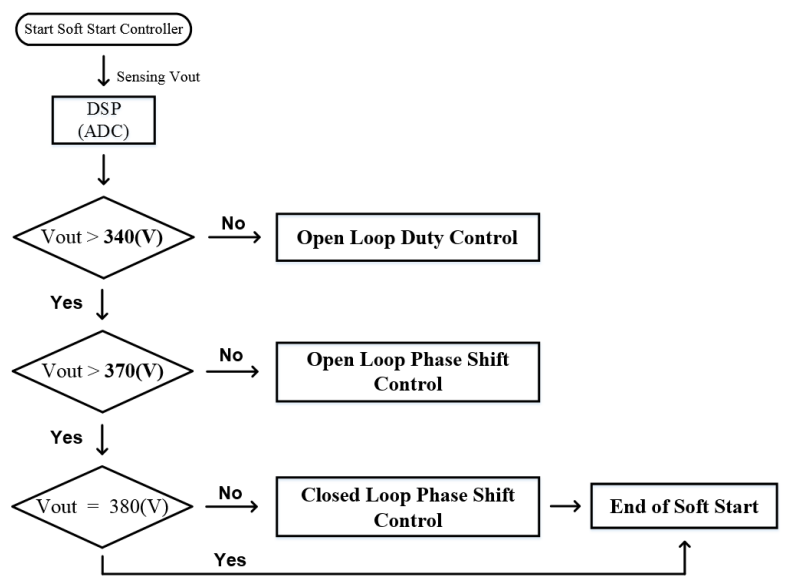

Fig. 9. The control diagram of the proposed three sequences soft start algorithm

the output capacitor regardless of the phase-shift condition in the DAB converter. It can be one of the reasons why the inrush current induces stress and heat, causing the power switches to breakdown in the DAB converter. Fig. 8 shows the simulation results using the PSIM software. It shows output voltage and input current waveforms at the coldstart state with only normal feedback control. When the output voltage rises quickly, the high inrush current fugitively flows through the switches. To reduce the stress from the inrush current, a soft start algorithm is required.

\subsection{Concepts of soft start algorithm}

To address the problems of the high inrush current during cold-start state, a soft start algorithm is proposed. As shown in Fig. 9, the algorithm is divided into the three sequence in terms of output voltage levels: an open loop duty control (OPDC), an open loop phase-shift control (OLPSC), and a closed loop phase-shift control (CLPSC). According to the output voltage level, proper control algorithms are selected to suppress the inrush current under the cold start sequence.

\subsection{Open loop phase control}

At the initial state, the OLDC is applied to the DAB 


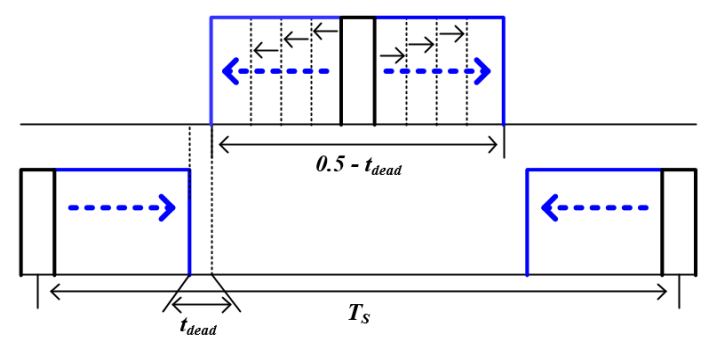

Fig. 10. Operational principle of the proposed open loop duty (OLD) algorithm

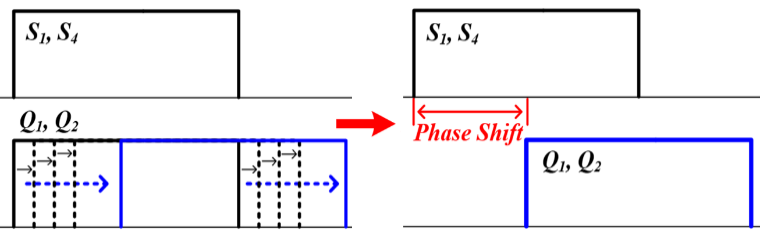

Fig. 11. Operational principle of the proposed open loop phase-shift (OLPS) algorithm

converter. Fig. 10 shows the process of the OLDC. At the beginning state, the duty ratio is not fixed as 0.5 but linearly increased from zero to 0.5 . This control is the same as the full-bridge converter PWM control manner, used to regulate the output voltage. The OLDC makes input current increase softly and reduces the peak value of the inrush current. Using the OLDC in the ideal case, the output voltage can effectively reach the reference voltage. However, due to parasitics, the target output voltage may not be reached. Therefore, to obtain the expected output voltage, the control mechanism should be improved.

The most suitable method is feedback control to regulate the output voltage to the reference. However, if the feedback control is achieved immediately after the OLDC, it can also cause high primary currents. This is because the phase of the gate signal, as a result of the OLDC, will be shifted in an instant. To prevent fast variations of the phase gap between the primary and secondary legs, the OLPSC is proposed. As shown in Fig. 11, the OLPSC algorithm makes the linear increase of the phase gap degree proportional to time duration. The OLPSC leads to reach the output voltage to the reference smoothly, and decreases peak inrush current simultaneously.

\subsection{Closed loop phase control}

After the overall open loop soft start controls are finished, the feedback controller starts to regulate the exact output voltage. The CLPSC is the normal feedback control algorithm of the DAB converter, which uses the difference between output and reference voltage. The key of the CLPSC in the proposed soft start algorithm is when the feedback controller starts in steady state operation. Fig. 12 shows the comparison of two CLPSC starting point cases.

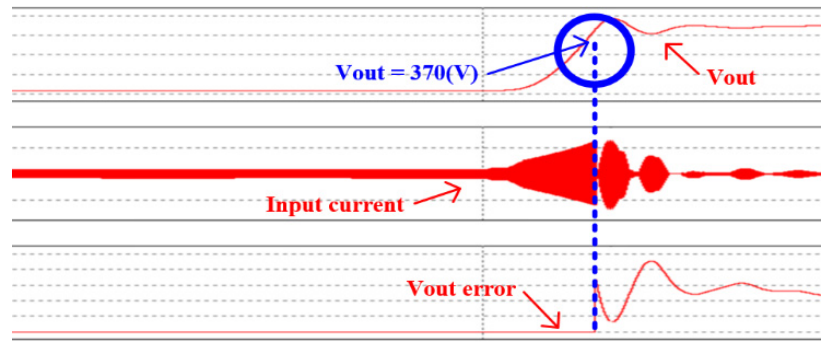

(a)

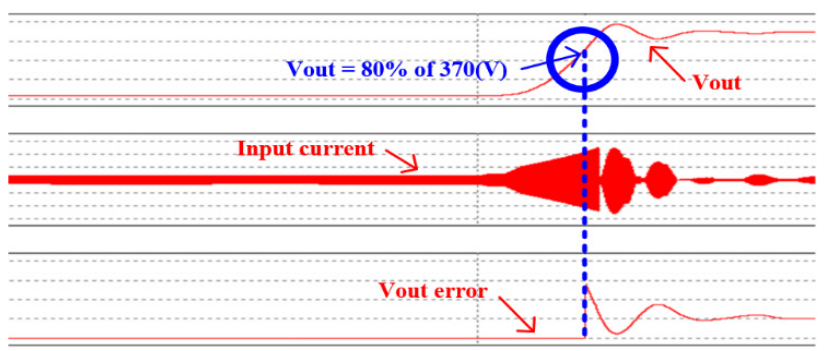

(b)

Fig. 12. Comparison of feedback control starting points: (a) After open loop control; (b) Before open loop control

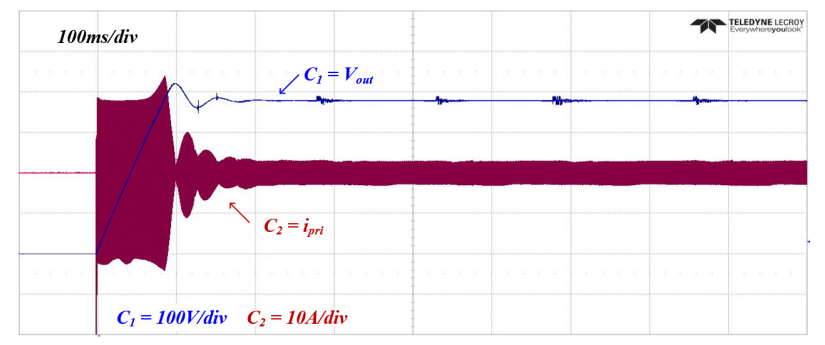

(a)

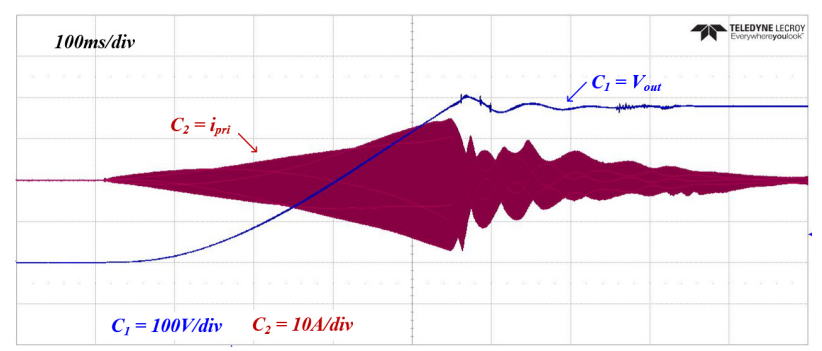

(b)

Fig. 13. Comparison of cold start waveforms: (a) Without the soft start algorithm; (b) With the soft start algorithm

Fig. 12 (a) indicates the waveform when the feedback control begins immediately after the overall open loop controls are ended. Fig. 12 (b) presents that the feedback control starts when the output voltage comes to $80 \%$ of the expected reference voltage. In case of Fig. 12 (b), the feedback controller accumulates and updates the errors before finishing the OLPSC. Although the latter has higher complexity and computational burden than the case of the 
former, the peak current in latter is reduced about $3 \%$.

Fig. 13 shows the comparison of the overall DAB converter cold start waveforms which are with/without the proposed soft start algorithm. Even though the settling time is extended, the RMS inrush current decreases from 14.45 A to 8.39 A when the soft start algorithm is implemented. In other words, the proposed soft start algorithm makes almost $41 \%$ reduction of RMS current. Also, the peak value of the inrush current decreases almost 8 A during the cold start.

Table 1. Design specification of the DAB converter

\begin{tabular}{c|c}
\hline Power & $3.3 \mathrm{~kW}$ \\
\hline Input Voltage & $380 \mathrm{~V}$ \\
\hline Output Voltage \& Current & $380 \mathrm{~V}, 8.7 \mathrm{~A}$ \\
\hline Frequency & $50 \mathrm{kHz}$ \\
\hline $\mathrm{d}($ degree$) / 180^{\circ}$ & $-0.48<\Phi<0.48$ \\
\hline Coupling Inductance & $102 \mu \mathrm{H}$ \\
\hline Dead time duration & $\mathrm{t}_{\min }=0.52 \mathrm{us}$ \\
\hline Length of air-gap & $1.53 \mathrm{~mm}$ \\
\hline Magnetizing inductance & $567.66 \mu \mathrm{H}$ \\
\hline Turn Ratio & $1: 1$ \\
\hline The Number of turns & $26 \mathrm{turns}$ \\
\hline B $_{\max }$ & $0.39 \mathrm{~T}$ \\
\hline Core & $\mathrm{EE} 6565 \mathrm{~S}$ \\
\hline
\end{tabular}

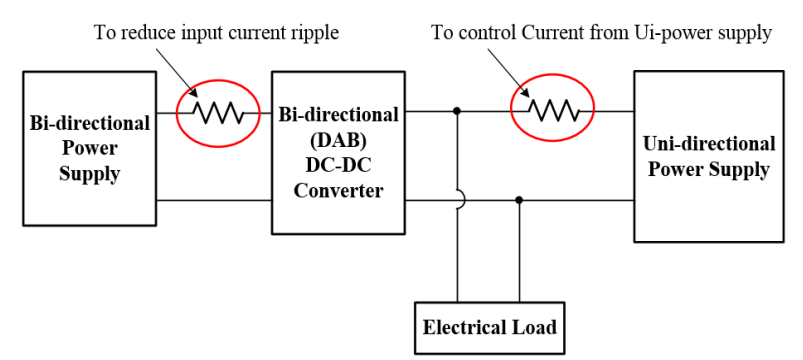

Fig. 14. Schematic of test condition in bi-directional power flows

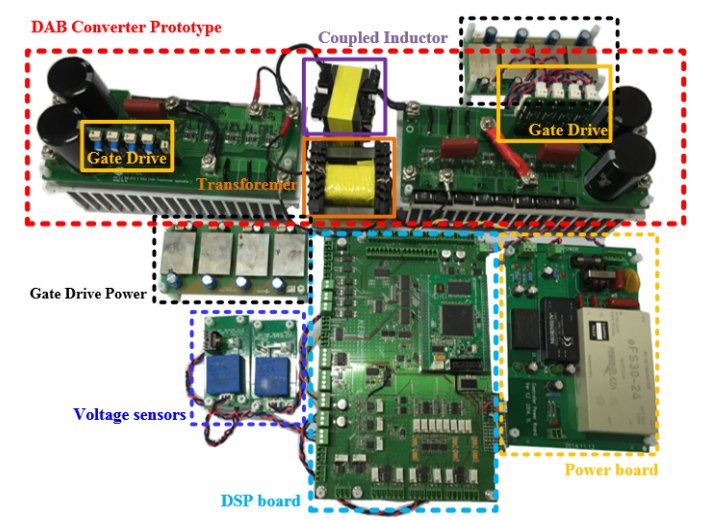

Fig. 15. Photograph of the $3.3 \mathrm{~kW}$ prototype DAB converter

\section{Experimental Results}

Table 1 indicates the specifications of the target experimental conditions and the prototype converter.
Following the suggested design guides, $t_{d}\left(=0.52 \mu_{\text {sec }}\right)$ and $L_{S}(=102 \mu \mathrm{H})$ are selected. Particularly, the coupling inductance is selected as $102 \mu \mathrm{H}$ based on the power rating and RMS current, as shown in Fig. 6 and Fig. 7. Fig. 14 shows the bi-directional power flows test condition. The resistor in front of the $\mathrm{DAB}$ converter is required to suppress the input current ripple. Another resistor which is connected to the output stage of the DAB converter is required to make a current source from a uni-directional power supply. Fig. 15 shows the $3.3 \mathrm{~kW}$ prototype DAB converter. It consists of a power stage board, a DSP board,

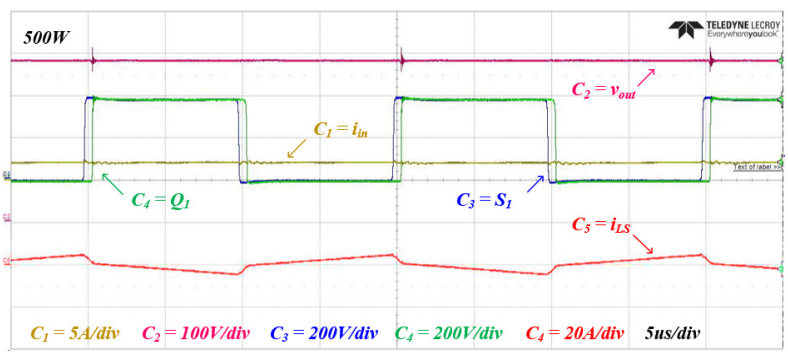

(a)

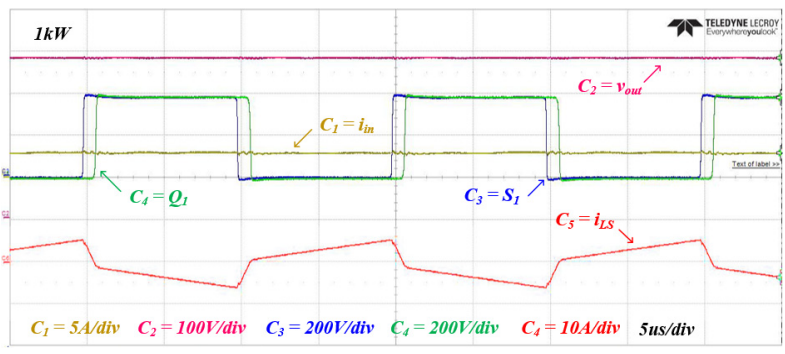

(b)

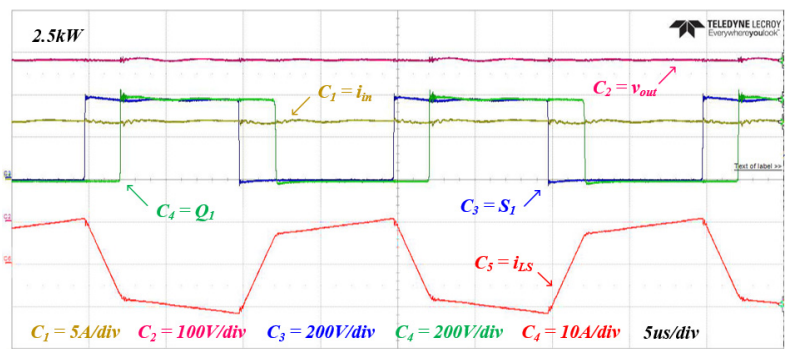

(c)

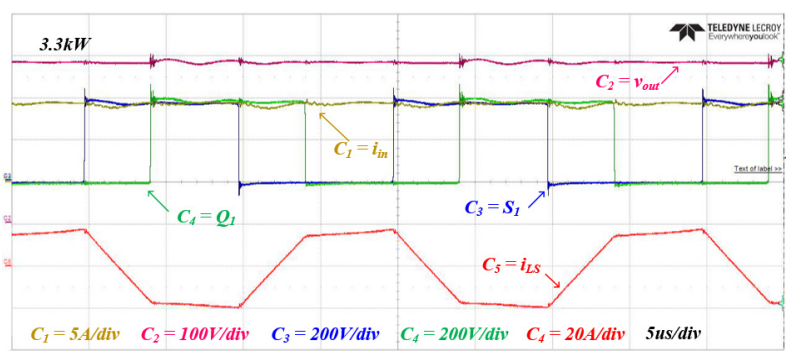

(d)

Fig. 16. Experiment waveforms of forward power flow according to power ratings: (a) $500 \mathrm{~W}$; (b) $1 \mathrm{~kW}$; (c) $2.5 \mathrm{~kW}$; (d) $3.3 \mathrm{~kW}$ 
voltage sensor boards, gate drivers, and auxiliary power supplies. The same PCB boards are applied on each transformer side due to the symmetric configuration. To get robustness against noise and disturbance, the high voltage ceramic capacitors are connected both high and low side legs. To suppress noise and stray inductance, the gate drives are located very close to the power switches.

Fig. 16 and 17 indicate the bi-directional power flows experimental results in forward and backward conditions, respectively. Each result is measured according to load variations from $500 \mathrm{~W}$ to $3.3 \mathrm{~kW}$. The input voltage is

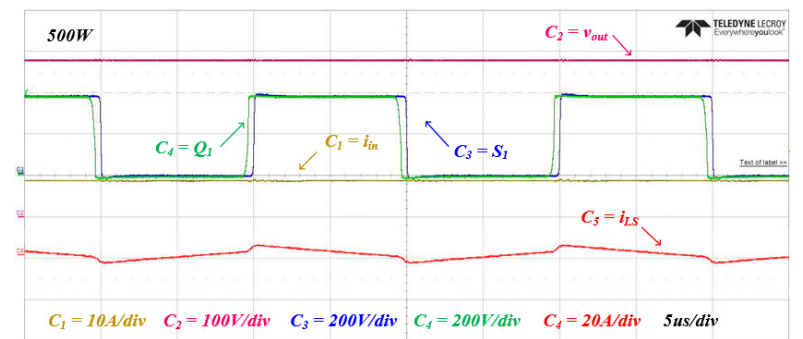

(a)

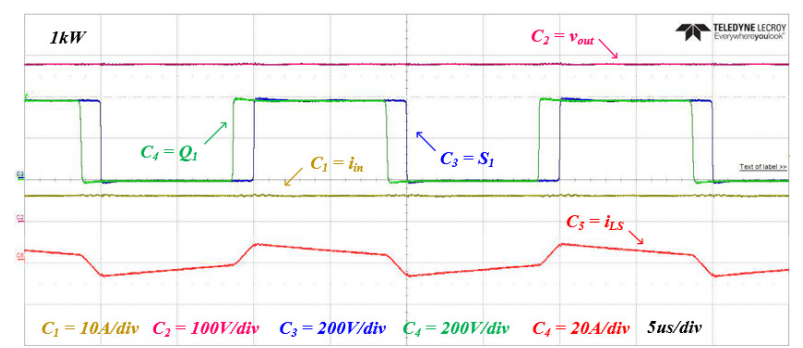

(b)

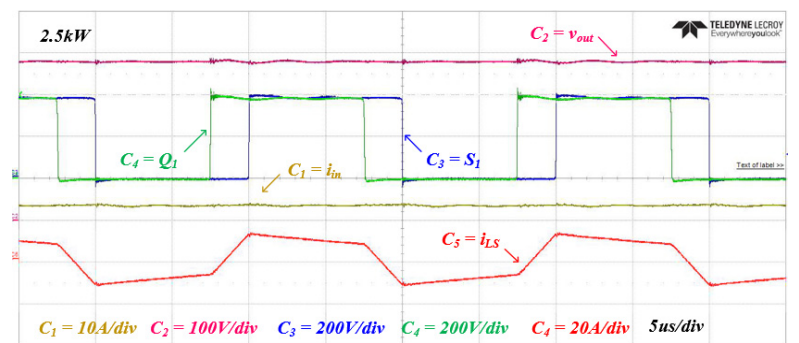

(c)

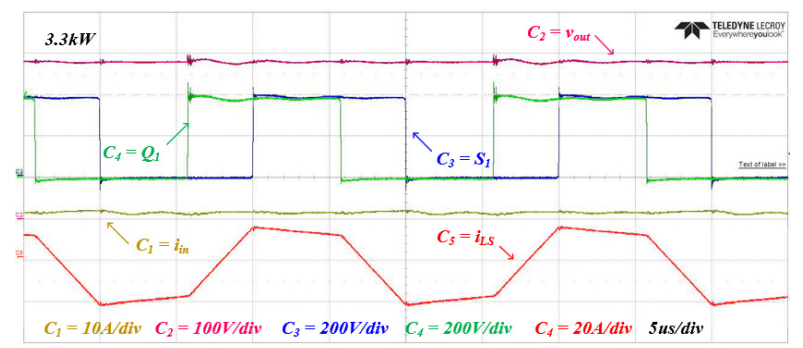

(d)

Fig. 17. Experimental waveforms of backward power flow according to power ratings: (a) $500 \mathrm{~W}$; (b) $1 \mathrm{~kW}$; (c) $2.5 \mathrm{~kW}$; (d) $3.3 \mathrm{~kW}$.

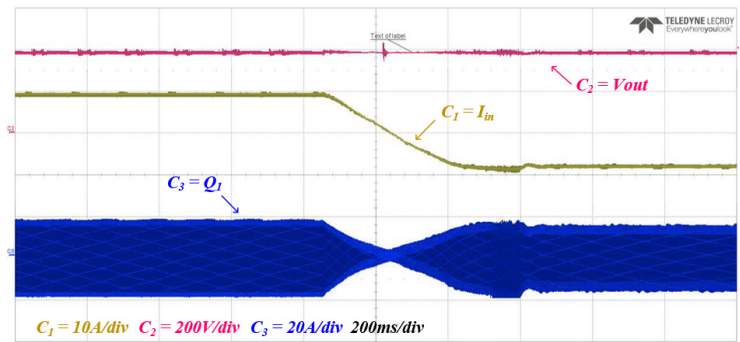

Fig. 18. Experimental waveform of bi-directional step load response from $3.3 \mathrm{~kW}$ to $-3.3 \mathrm{~kW}$

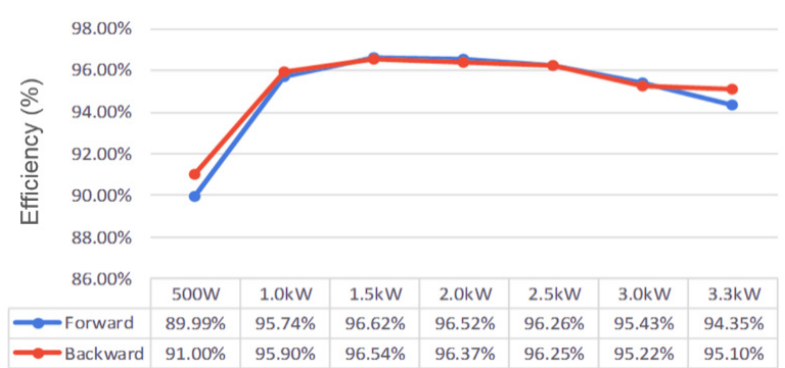

Fig. 19. Comparison of power conversion efficiency between forward and backward power flows

fixed to $380 \mathrm{~V}$, and the target output voltage is regulated to $380 \mathrm{~V}$. As shown in Fig. 16 and 17, phase gap between the primary and secondary legs signify the amount of transferred power. At the $3.3 \mathrm{~kW}$ condition in the forward power flow, th,e output voltage ripple is only about $2.5 \%$ of reference. Fig. 18 shows the bi-directional step load response from the forward direction of $3.3 \mathrm{~kW}$ to the backward direction of $-3.3 \mathrm{~kW}$. Although the dynamic step load variation is $6.6 \mathrm{~kW}$ with changing power flow directions, the output voltage is regulated well.

Fig. 19 indicates the efficiency curves of the prototype $\mathrm{DAB}$ converter according to the load variation from $500 \mathrm{~W}$ to $3.3 \mathrm{~kW}$. At the $1.5 \mathrm{~kW}$ condition, the highest efficiency is measured as $96.62 \%$ and $96.54 \%$ with forward and backward power flow, respectively. In addition, at the full load conditions with forward and backward power flows, the power conversion efficiency is measured as $94.35 \%$ and $95.10 \%$, respectively. At light load conditions, the efficiency is lower than the heavy load cases since the conduction loss due to the circulating current passing through the MOSFET, transformer and inductor is more significant. At the full load conditions, the power conversion efficiency also decreases since the conduction loss is increased by higher load current.

\section{Conclusion}

This paper presents an accurate DAB converter design methodology for SST applications. To ensure the maximum soft switching capability, and improve the overall efficiency, the proper design guidelines for the coupling inductance 
and the dead time duration are presented by using detailed analysis of the DAB converter. In addition, by applying the effective soft start algorithm, the inrush current through the switches is drastically suppressed at the startup state of the DAB converter. A $3.3 \mathrm{~kW}$ prototype DAB converter is designed and implemented to verify the bidirectional operation. The experimental results such as the fast bi-directional step load response with output voltage regulation and high power conversion efficiency demonstrate the practical feasibility of the proposed DAB converter design.

\section{Acknowledgement}

This work was supported by the 2013 Future-Strategic Fund (Project No. 1.130034) of UNIST (Ulsan National Institute of Science and Technology).

\section{References}

[1] P. Chiradeja and R. Ramakumar, "An approach to quantify the technical benefits of distributed generation," IEEE Transactions. Energy Convers., vol. 19, no. 4, pp. 764-773, Dec. 2004.

[2] H. Bevrani, A. Ghosh, and G. Ledwich, "Renewable energy sources and frequency regulation: Survey and new perspectives," IET Renew. Power Gener., vol. 4, no. 5, pp. 438-457, Sep. 2000.

[3] J. M. Carrasco, L. G. Franquelo, J. T. Bialasiewcz, E. Galvan, R. C. P. Guisado, M. A. M. Prats, J. I. Leon, and N. Moreno-Alfonso, "Power-electronic systems for the grid integration of renewable energy sources: A survey," IEEE Trans. Ind. Appl., vol. 53, no. 4, pp. 1002-1016, Jul./Aug. 2006.

[4] H. J. Choi, H. P. Park, and J. H. Jung, "Design Methodology of dual active bridge converter for solid state transformer application in smart grid" Proc. IEEE ECCE Asia Conf., pp. 196-201, Jun. 2015.

[5] X. Yu, X. She, X. Zhou, and A. Q. Huang, "Power management for DC microgrid enabled by solid-state transformer," IEEE Trans. Smart Grid, vol. 5, no. 2, pp. 954-965, Mar. 2014.

[6] M. G. Kanabar, I. Voloh, and D. McGinn, "A Review of Smart Grid Standards for Protection, Control, and Monitoring Applications," the 2012 65th Annual Conference for Protective Relay Engineers, Texas (USA), Apr. 2012.

[7] X. She, R. Burgos, G. Y. Wang, F. Wang, and A. Q. Huang, "Review of Solid State Transformer in the Distribution System: From Components to Field Application", Energy Conversion Congress and Exposition (ECCE-Carolina 2012), Carolina (USA), Sep. 2012.
[8] Y. Du, S. Baek, S. Bhattacharya, and A. Q. Huang, "High-voltage high frequency transformer design for a $7.2 \mathrm{kV}$ to $120 \mathrm{~V} / 240 \mathrm{~V} 20 \mathrm{kVA}$ solid state transformer," the 36th Annu. Conf. IEEE Ind. Electron. Soc (IECON-Arizona 2010), Arizona (USA), Nov. 2010.

[9] L. H. Mweene, C. A. Wright, and M. F. Schlecht, "A $1 \mathrm{~kW}, 500 \mathrm{kHz}$ front-end converter for a distributed power supply system", IEEE Transactions. Power Electron, Vol. 6, No. 3, pp. 398 407, Jul. 1991.

[10] B. Zhao, Q. Yu, and W. Sun, "Extended-phase-shift control of isolated bidirectional DC-DC converter for power distribution in microgrid," IEEE Trans. Power Electron., vol. 27, no. 11, pp. 4667-4680, Nov. 2012.

[11] M. N. Kheraluwala, R. W. Gascoigne, D. M. Divan, and E. D. Baumann, "Performance characterization of a high-power dual active bridge," IEEE Trans. Ind. Appl., vol. 28, no. 6, pp. 1294-1301, 1992.

[12] A. Alonso, J. Sebastian, D. Lamar. M. Hernando, and A. Vazquez, "An overall study of a dual active bridge for bidirectional dc/dc conversion", Energy Conversion Congress and Exposition (ECCE-Georgia 2010), Georgia (USA), Sep. 2010.

[13] C. Mi, H. Bai, C. Wang, and S. Gargies, "Operation, design and control of dual h-bridge-based isolated bidirectional dc-dc converter," Power Electronics, IET, vol. 1, no. 4, pp. 507-517, Dec. 2008.

[14] R. T. Naayagi, A. J. Forsyth, and R. Shuttleworth, "High-power bidirectional DC-DC converter for aerospace applications," IEEE Trans. Power Electron., vol. 27, no. 11, pp. 4366-4379, Nov. 2012

[15] S. Inoue and H. Akagi, "A bidirectional DC-DC converter for an energy storage system with galvanic isolation", IEEE Trans. Power Electron., Vol. 22. No. 6, pp.2299-2306, Nov. 2007.

[16] J. H. Jung, H. S. Kim, M. H. Ryu, and J. W. Baek, "Design methodology of bidirectional CLLC resonant converter for high frequency isolation of DC distribution systems," IEEE Trans. Power Electronics, Vol. 28, No. 4, pp. 1741-1755, Apr. 2013.

[17] D. K. Jeong, M. H. Ryu, H. G Kim, H. J. Kim, "Optimized design of bi-directional dual active bridge converter for low-voltage battery charger," Journal of Power Electronics, vol. 14. No. 3, pp. 468-477, May 2014.

[18] H. Wen, B. Su, "Reactive Power and Soft-Switching Capability Analysis of Dual-Active-Bridge DC-DC Converters with Dual-Phase-Shift Control", Journal of Power Electronics, vol. 15. No, 1, pp. 18-30, Jan 2015.

[19] R. W. Erickson and D. Maksimovic, Fundamentals of Power Electronics, 2nd ed. Norwell, MA: Kluwer, 2001, p. 500. 


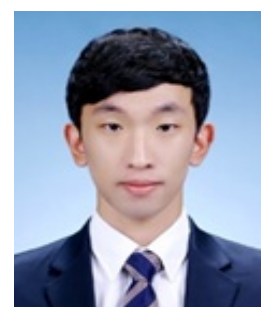

Hyun-Jun Choi He was born in Busan, Korea, in 1989. He received B.S degree from Seoul National University of Science and Technology, Seoul, Korea in 2013. He is currently working toward the Ph.D. degree with the School of Electrical and Computer Engineering, Ulsan National Institute of Science and Technology (UNIST), Ulsan, Korea. His research interests include bi-directional converter, and digital control algorithm of bi-directional power flows.

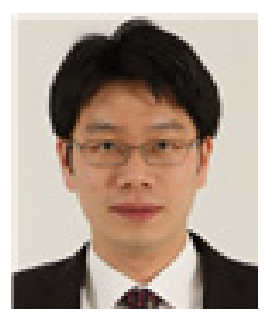

Jee-Hoon Jung He was born in Suwan, Korea, in 1977. He received the B.S., M.S., and Ph.D. degree from Pohang University of Science and Technology (POSTECH), Pohang, Korea, in 2000, 2002, and 2006, respectively. From 2006 to 2009 , he was a Senior Research Engineer with the Digital Printing Division, Samsung Electronics Company Ltd., Suwon, Korea. From 2009 to 2010, he was a Postdoctoral Research Associate with the Department of Electrical and Computer Engineering, Texas A\&M University of Qatar (TAMUQ), Doha, Qatar. From 2011 to 2012, he was a Senior Researcher with the Power Conversion and Control Research Center, HVDC Research Division, Korea Electro Technology Research Institute (KERI), Changwon, Korea. Since 2013, he has been an Assistant Professor with the School of Electrical and Computer Engineering, Ulsan National Institute of Science and Technology (UNIST), Ulsan, Korea. His research interests include dc-dc converters, switched-mode power supplies, motor drives and diagnosis systems, digital control and signal processing algorithms, digitally controlled power electronics, power conversion for renewable energy, and real-time and power hardwarein-the-loop simulations (HILS) of renewable energy sources. Recently, he has been researching high frequency power converters using wide bandgap devices, smart power transformers for smart grid, multi-level converter and power control algorithms for DC microgrid, control and power HILS for electric ship's power network analysis, and wireless power transfer techniques for electric vehicle and home appliance applications.

Dr. Jung is a Senior Member of the IEEE Industrial Electronics Society, the IEEE Power Electronics Society, the IEEE Industry Applications Society, and the IEEE Power and Energy Society, and he is an Associated Editor of Journal of Power Electronics and a member of the Editorial Committee of the Korea Institute of Power Electronics. 